\title{
Preparation of Fullerendione through Oxidation of Vicinal Fullerendiol and Intramolecular Coupling of the Dione to Form Hemiketal/Ketal Moieties
}

\author{
Shaohua Huang, ${ }^{a}$ Fudong Wang, ${ }^{a}$ Liangbing Gan, ${ }^{* a, b}$ Gu Yuan, ${ }^{c}$ Jiang Zhou, ${ }^{c}$ Shiwei Zhang $*^{d}$ \\ ${ }^{a}$ Key Laboratory of Bioorganic Chemistry and Molecular Engineering of the Ministry of Education, College of Chemistry, \\ Peking University, Beijing 100871, China; ${ }^{b}$ State Key Laboratory of Organometallic Chemistry, Shanghai Institute of \\ Organic Chemistry, Chinese Academy of Sciences, 354 Fenglin Lu, Shanghai 200032, China; ${ }^{\mathrm{c} C o l l e g e}$ of Chemistry and \\ Molecular Engineering, Peking University, Beijing 100871; ${ }^{\mathrm{d}}$ State Key Laboratory for Structural Chemistry of Unstable \& \\ Stable Species, Beijing 100871, China
}

\section{Experimental procedure and characterization data}

All the reagents were used as received. $\mathrm{CH}_{2} \mathrm{Cl}_{2}$ used for reactions was distilled from $\mathrm{P}_{2} \mathrm{O}_{5}$. Other solvents were used as received. The reactions were carried out under atmosphere. Compound 1 and anhydrous $\mathrm{H}_{2} \mathrm{O}_{2}$ in $\mathrm{CH}_{2} \mathrm{Cl}_{2}$ used for the preparation of 4c were prepared as in Huang, S. H.; Xiao, Z.; Wang, F. D.; Zhou, J.; Yuan, G.; Zhang, S. W.; Chen, Z. F.; Thiel, W.; Schleyer, P. R.; Zhang, X.; Hu, X. Q.; Chen, B. C.; Gan, L. B. Chem. Eur. J. 2005, 11, 5449. Caution: a large amount of peroxides are involved in some of the reactions, care must be taken to avoid possible explosion.

Preparation of fullerene diol 2. $\left(\mathrm{C}_{6} \mathrm{~F}_{5}\right)_{3} \mathrm{~B}(10 \mathrm{mg}, 0.019 \mathrm{mmol}$ was added to a solution of $\mathbf{1}(40 \mathrm{mg}, 0.037 \mathrm{mmol})$ in $5 \mathrm{ml}$ commercial $\mathrm{CH}_{2} \mathrm{Cl}_{2}$ (used directly). The mixture was stirred in dark at room temperature. Progress of the reaction was monitored by TLC analysis. Five drops of $2 \mathrm{M}$ aqueous $\mathrm{HCl}$ were added to the mixture when the product reached its maximum. The organic layer was separated, dried with $\mathrm{Na}_{2} \mathrm{SO}_{4}$, filtered. The solvent was removed. The residue was chromatographed with benzene/petroleum ether $\left(60-90{ }^{\circ} \mathrm{C}\right) /$ ethyl acetate $(10 / 10 / 1)$ as eluent to give 2 as an orange band (30 $\mathrm{mg}, 74 \%)$.

This is an improved method. Characterization data of 2 can be found in Huang, S. H.; Xiao, Z.; Wang, F. D.; Gan, L. B.; Zhang, X.; Hu, X. Q.; Zhang, S. W.; Lu, M. J.; Pan, J. Q.; Xu, L. J. Org. Chem. 2004, 69, 2442.

Preparation of fullerene dione 3. $\mathrm{PhI}(\mathrm{OAc})_{2}(17 \mathrm{mg}, 0.053 \mathrm{mmol})$ was added to a solution of $2(30 \mathrm{mg}, 0.027 \mathrm{mmol})$ in $6 \mathrm{ml}$ benzene. The mixture was stirred in dark at $35^{\circ} \mathrm{C}$. Progress of the reaction was monitored by TLC analysis. After 1.5 hour, the mixture was chromatographied with benzene/petroleum ether $\left(60-90^{\circ} \mathrm{C}\right) / \mathrm{ethyl}$ acetate $(10 / 10 / 1)$ as eluent to give 3 as the first band ( $24 \mathrm{mg}, 80 \%$ ), then followed by unreacted 2 (trace) as the second band.

${ }^{1} \mathrm{H} \mathrm{NMR}\left(\mathrm{CDCl}_{3}, 300 \mathrm{MHz}\right): \delta 1.45(\mathrm{~s}, 18 \mathrm{H}), 1.41 \mathrm{ppm}(\mathrm{s}, 18 \mathrm{H}) ;{ }^{13} \mathrm{C} \mathrm{NMR}\left(\mathrm{CDCl}_{3}, 75 \mathrm{MHz}\right.$, all signals represent $2 \mathrm{C}$ except noted): $\delta 196.88$ (1C, C=O), 196.64 (1C, C=O), 152.51, 149.70, 149.28 (3C), 148.92 (1C), 148.85, 148.68, 148.63, $148.54,148.01,147.39,147.25,147.01,146.64,146.42,145.05,145.01,144.90,144.80,144.00,143.29,143.20,143.01$, $141.52,141.50,141.21,139.20,131.48,88.37,82.39\left(2 \mathrm{C}\left(\mathrm{CH}_{3}\right)_{3}\right), 82.20\left(2 \mathrm{C}\left(\mathrm{CH}_{3}\right)_{3}\right), 78.23,26.62\left(6 \mathrm{CH}_{3}\right), 26.55\left(6 \mathrm{CH}_{3}\right)$ ppm; FTIR (microscope): 2979, 2928, 2868, 1751, 1472, 1456, 1387, 1364, 1243, 1192, 1102, 1077, 1044, 1023, 1009, 869, 784, $758 \mathrm{~cm}^{-1}$; ESI-MS: m/z (\%): $1126(100)[\mathrm{M}+\mathrm{OH}+\mathrm{H}]$; calcd for $\mathrm{C}_{76} \mathrm{H}_{36} \mathrm{O}_{10}: 1108$.

General procedure for preparation of hemiketal/ketal derivatives 4. Excess oxygen nucleophile and Lewis acid were added to a solution of $\mathbf{3}$ in $\mathrm{CH}_{2} \mathrm{Cl}_{2}$. The mixture was stirred in dark at room temperature. Progress of the reaction was monitored by TLC analysis. When the product reached its maximum, $2 \mathrm{ml}$ of $2 \mathrm{M}$ aqueous $\mathrm{HCl}$ was added to the mixture. The organic layer was separated, dried with $\mathrm{Na}_{2} \mathrm{SO}_{4}$, filtered. The solvent was removed. The residue was chromatographed 
with benzene/petroleum ether $\left(60-90{ }^{\circ} \mathrm{C}\right) / \mathrm{ethyl}$ acetate $(10 / 10 / 1)$ as eluent to afford the desired product. The reaction time and the yield for each individual nucleophile are listed in Table 1 (usually about $40 \mathrm{mg}$ of $\mathbf{3}$ was used in each run).

Table 1. Yields and conditions for the preparation of compounds 4

$\begin{array}{lllcc}\text { Nucleophile } & \text { Lewis Acid } & \text { Time } & \text { Product } & \text { Yield } \\ \mathrm{H}_{2} \mathrm{O} \text { (not added, from the reaction media) } & \mathrm{BF}_{3} \cdot \mathrm{Et}_{2} \mathrm{O}(1 \text { eq.) } & 5 \text { min. } & \mathbf{4 a} & 62 \% \\ \mathrm{H}_{2} \mathrm{O} \text { (not added, from the reaction media) } & \mathrm{Me}_{3} \mathrm{O} \cdot \mathrm{BF}_{4}(6 \text { eq.) } & 5 \text { min. } & \mathbf{4 a} & 49 \% \\ \mathrm{MeOH}(300 \text { eq) } & \mathrm{BF}_{3} \cdot \mathrm{Et}_{2} \mathrm{O} \text { (7 eq.) } & 3 \mathrm{~h} & \mathbf{4 b} & 73 \% \\ \mathrm{H}_{2} \mathrm{O}_{2} \text { (in } \mathrm{CH}_{2} \mathrm{Cl}_{2}, 80 \text { eq) } & \mathrm{BF}_{3} \cdot \mathrm{Et}_{2} \mathrm{O}(1 \text { eq.) } & 2 \text { min. } & \mathbf{4 c} & 65 \% \\ { }^{t} \mathrm{BuOOH} \text { (in decane (5.5 mol/L), 10 eq) } & \mathrm{BF}_{3} \cdot \mathrm{Et}_{2} \mathrm{O} \text { (1 eq.) } & 5 \text { min. } & \mathbf{4 d} & 65 \%\end{array}$

Spectroscopic data of $4 \mathbf{4 a} .{ }^{1} \mathrm{H}$ NMR $\left(\mathrm{CDCl}_{3}, 400 \mathrm{MHz}\right): \delta 4.98$ (s, OH), 4.51 (s, OH), 1.47 (s, 18H), $1.43 \mathrm{ppm}(\mathrm{s}, 18 \mathrm{H})$;

${ }^{13} \mathrm{C} \mathrm{NMR}\left(\mathrm{CDCl}_{3}, 100 \mathrm{MHz}\right.$, all signals represent 2C except noted): $\delta 162.10,149.70,148.91,148.79,148.68$ (1C), 148.51, 148.41, 148.29, 148.18 (1C), 148.12, 147.06, 147.03, 147.02, 146.96, 146.90, 146.70, 145.73, 145.31, 144.84, 144.78, $144.02,143.62,143.09,142.95,141.94,141.45,139.82,131.66,106.40(1 \mathrm{C}), 102.51(1 \mathrm{C}), 88.14,82.36\left(2 \mathrm{C}\left(\mathrm{CH}_{3}\right)_{3}\right), 81.87$ $\left(2 \mathrm{C}\left(\mathrm{CH}_{3}\right)_{3}\right), 79.05,26.82\left(6 \mathrm{CH}_{3}\right), 26.77 \mathrm{ppm}\left(6 \mathrm{CH}_{3}\right)$; FTIR (microscope): 3539, 2978, 2929, 2869, 1473, 1456, 1388, 1364, 1243, 1193, 1158, 1106, 1086, 1056, 1019, 871, $753 \mathrm{~cm}^{-1}$; ESI-MS: m/z (\%): 1144 (100) [M+OH+H]; calcd for $\mathrm{C}_{76} \mathrm{H}_{38} \mathrm{O}_{11}$ : 1126.

Spectroscopic data of $\mathbf{4 b} .{ }^{1} \mathrm{H}$ NMR $\left(\mathrm{CDCl}_{3}, 400 \mathrm{MHz}\right): \delta 4.94$ (s, OH), $3.87(\mathrm{~s}, 3 \mathrm{H}), 1.47(\mathrm{~s}, 18 \mathrm{H}), 1.44 \mathrm{ppm}(\mathrm{s}, 18 \mathrm{H}) ;{ }^{13} \mathrm{C}$ $\mathrm{NMR}\left(\mathrm{CDCl}_{3}, 100 \mathrm{MHz}\right.$, all signals represent 2C except noted): $\delta 162.61,149.70,148.93,148.78,148.63$ (1C), 148.46, 148.43, 148.29, 148.14 (1C), 148.05, 147.07, 147.05, 146.93, 146.91, 146.71 (4C), 145.76, 144.98, 144.73, 144.71, 144.01, $143.57,143.10,142.92,141.94,141.47,139.93,133.44,106.38$ (1C), $105.62(1 \mathrm{C}), 88.05,82.21\left(2 \mathrm{C}\left(\mathrm{CH}_{3}\right)_{3}\right), 81.81$ $\left(2 \mathrm{C}\left(\mathrm{CH}_{3}\right)_{3}\right), 79.09,53.95\left(1 \mathrm{CH}_{3}\right), 26.75 \mathrm{ppm}\left(12 \mathrm{CH}_{3}\right)$; FTIR (microscope): 3537, 2978, 2932, 2873, 1473, 1455, 1388, 1364, 1247, 1193, 1162, 1109, 1088, 1056, 1043, 1020, 1004, 909, 872, $734 \mathrm{~cm}^{-1}$; ESI-MS: m/z (\%): 1158 (100) $[\mathrm{M}+\mathrm{OH}+\mathrm{H}]$; calcd for $\mathrm{C}_{77} \mathrm{H}_{40} \mathrm{O}_{11}: 1140$.

Spectroscopic data of 4c. ${ }^{1} \mathrm{H}$ NMR $\left(\mathrm{CDCl}_{3}, 300 \mathrm{MHz}\right): \delta 9.50(\mathrm{OOH}), 5.23$ (broad, $\left.\mathrm{OH}\right), 1.48$ (s, 18H), $1.42 \mathrm{ppm}(\mathrm{s}$, $18 \mathrm{H}) ;{ }^{13} \mathrm{C} \mathrm{NMR}\left(\mathrm{CDCl}_{3}, 75 \mathrm{MHz}\right.$, all signals represent $2 \mathrm{C}$ except noted): $\delta 162.10,149.69,148.90,148.88,148.65,148.57$, 148.41, 148.22, 148.08 (3C), 147.16, 146.92, 146.81 (4C), 146.60, 145.69, 145.51, 144.82, 144.60,144.08 (1C), 144.06, $143.55,143.04,143.02,141.79,141.67,139.79,134.32,109.29(1 \mathrm{C}), 106.55(1 \mathrm{C}), 88.11,82.33\left(2 \mathrm{C}\left(\mathrm{CH}_{3}\right)_{3}\right), 81.93$ (2C $\left.\left(\mathrm{CH}_{3}\right)_{3}\right), 79.00,26.74$ ppm (12 $\left.\mathrm{CH}_{3}\right)$; FTIR (microscope): 3535, 2978, 2931, 2870, 1474, 1455, 1388, 1364, 1244, 1192, 1162, 1104, 1085, 1016, 869, $753 \mathrm{~cm}^{-1}$; ESI-MS: m/z (\%): 1160 (100) [M+OH+H]; calcd for $\mathrm{C}_{76} \mathrm{H}_{38} \mathrm{O}_{12}: 1142$

Spectroscopic data of 4d. ${ }^{1} \mathrm{H}$ NMR $\left(\mathrm{CDCl}_{3}, 400 \mathrm{MHz}\right): \delta 4.86(\mathrm{~s}, \mathrm{OH}), 1.47(\mathrm{~s}, 18 \mathrm{H}), 1.42 \mathrm{ppm}(\mathrm{s}, 18 \mathrm{H}), 1.37(\mathrm{~s}, 9 \mathrm{H}) ;{ }^{13} \mathrm{C}$ NMR $\left(\mathrm{CDCl}_{3}, 100 \mathrm{MHz}\right.$, all signals represent 2C except noted): $\delta 162.13,149.64,148.93,148.84,148.53$ (1C), 148.43, 148.33, 148.22, 148.05 (1C), 147.95, 147.30, 147.18, 147.08, 146.71, 146.58, 145.71, 145.59, 145.11, 144.71, 144.64, $143.97,143.59,142.92,142.74,141.87,141.37,139.87,133.58,107.79(1 \mathrm{C}), 106.42(1 \mathrm{C}), 88.14,82.01\left(2 C\left(\mathrm{CH}_{3}\right)_{3}\right), 81.94$ $\left(C\left(\mathrm{CH}_{3}\right)_{3}\right), 81.78\left(2 \mathrm{C}\left(\mathrm{CH}_{3}\right)_{3}\right), 79.01,27.00\left(3 \mathrm{CH}_{3}\right), 26.76 \mathrm{ppm}\left(6 \mathrm{CH}_{3}\right), 26.70\left(6 \mathrm{CH}_{3}\right)$; FTIR (microscope): 3541, 2979, 2931, 2871, 1474, 1455, 1388, 1364, 1248, 1193, 1163, 1108, 1086, 1016, 871, $757 \mathrm{~cm}^{-1}$; ESI-MS: m/z (\%): 1216 (100) $[\mathrm{M}+\mathrm{OH}+\mathrm{H}]$; calcd for $\mathrm{C}_{80} \mathrm{H}_{46} \mathrm{O}_{12}: 1198$. 
CCDC- 284232 contains the supplementary crystallographic data for $\mathbf{4 d}$. The crystallographic data can be obtained free of charge via www.ccdc.cam.ac.uk/consts/retrieving.html (or from the Cambridge Crystallographic Data Centre, 12 Union Road, Cambridge CB12 1EZ, UK; fax: (+44)1223-366-033; or e-mail: deposit@ccdc.cam.ac.uk.

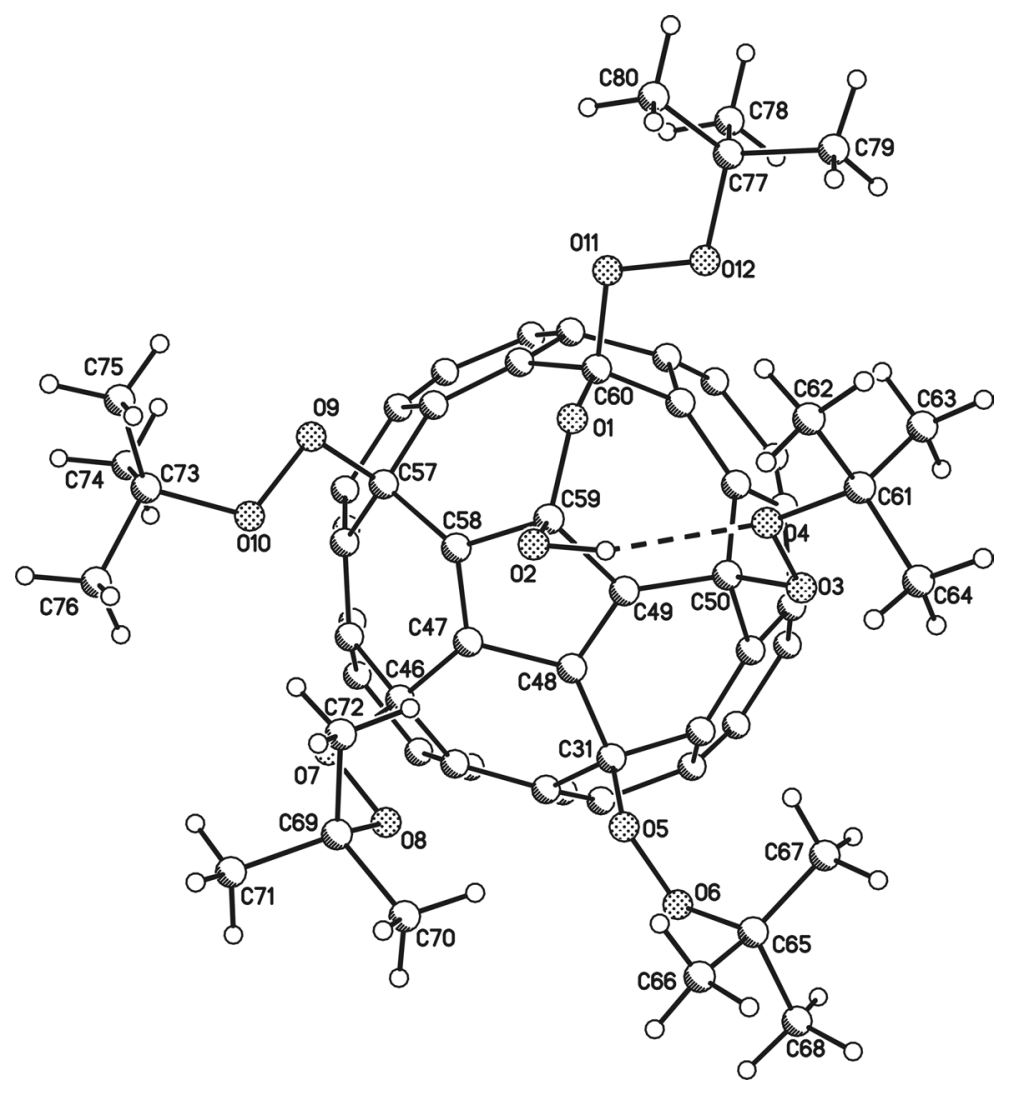

Single Crystal structure of $\mathbf{4 d}$ 


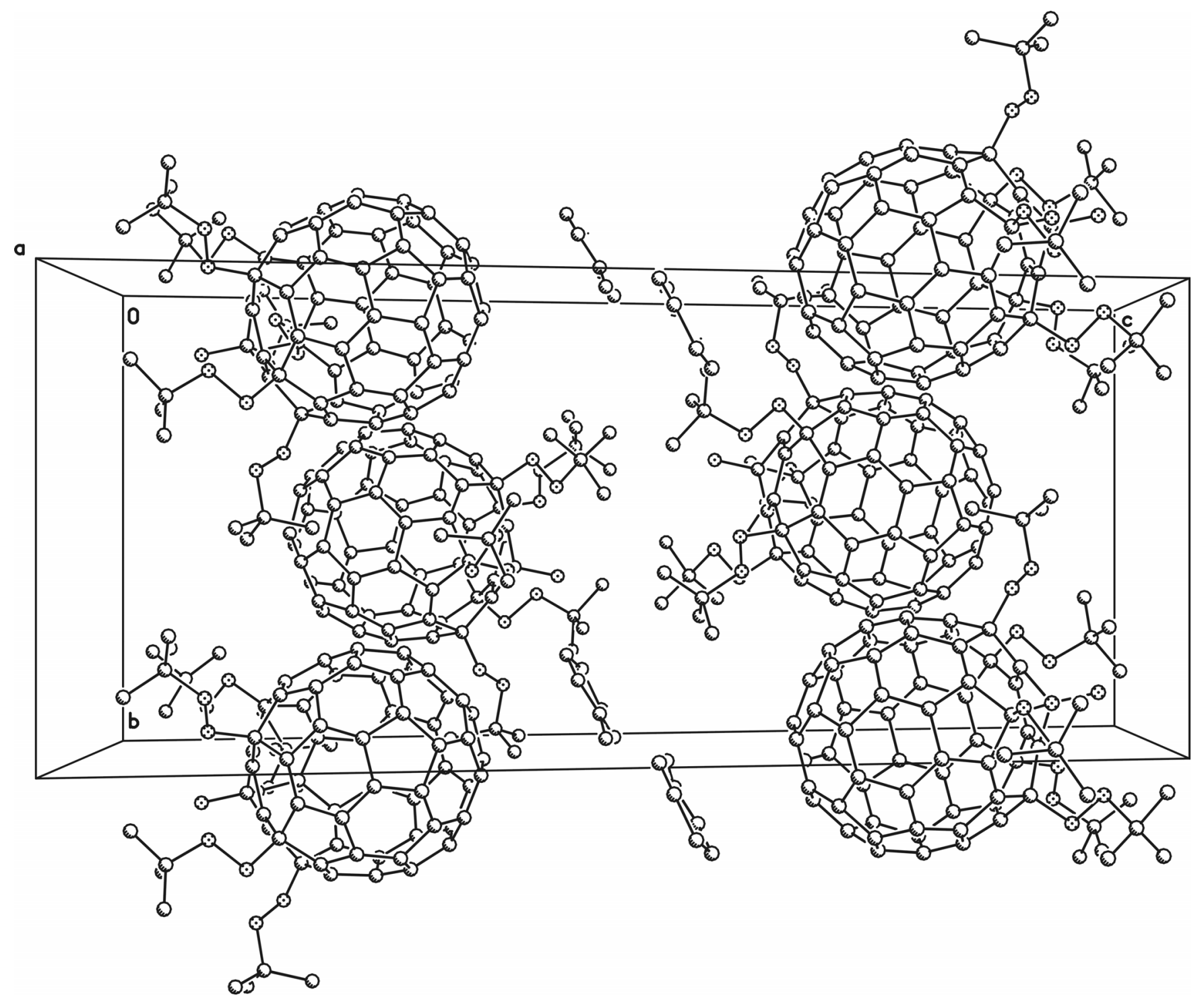

Single crystal unit cell of $\mathbf{4 d}$ 


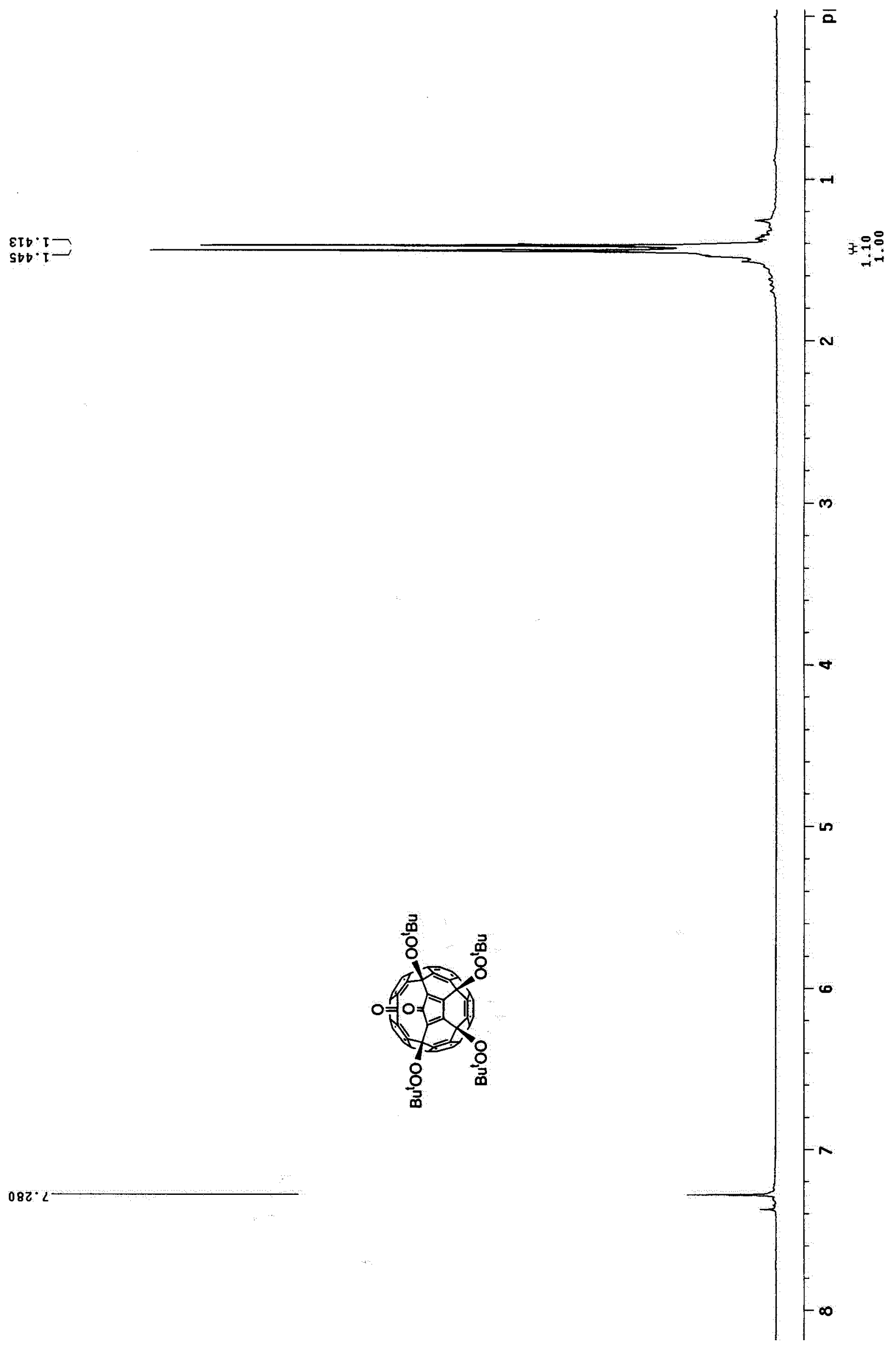




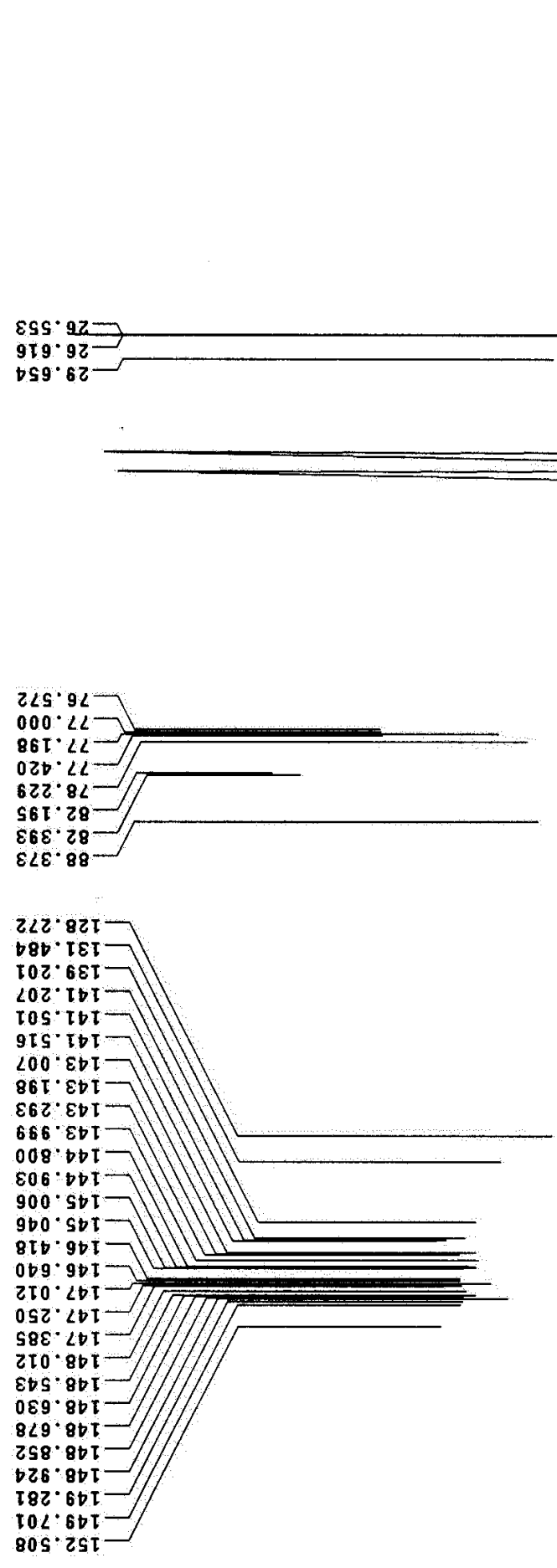

869.965

โ88.96
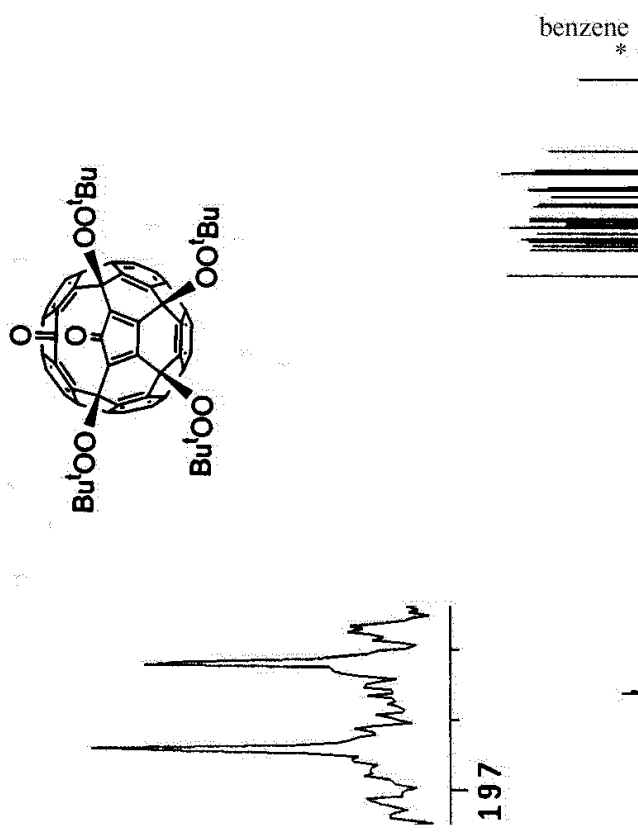


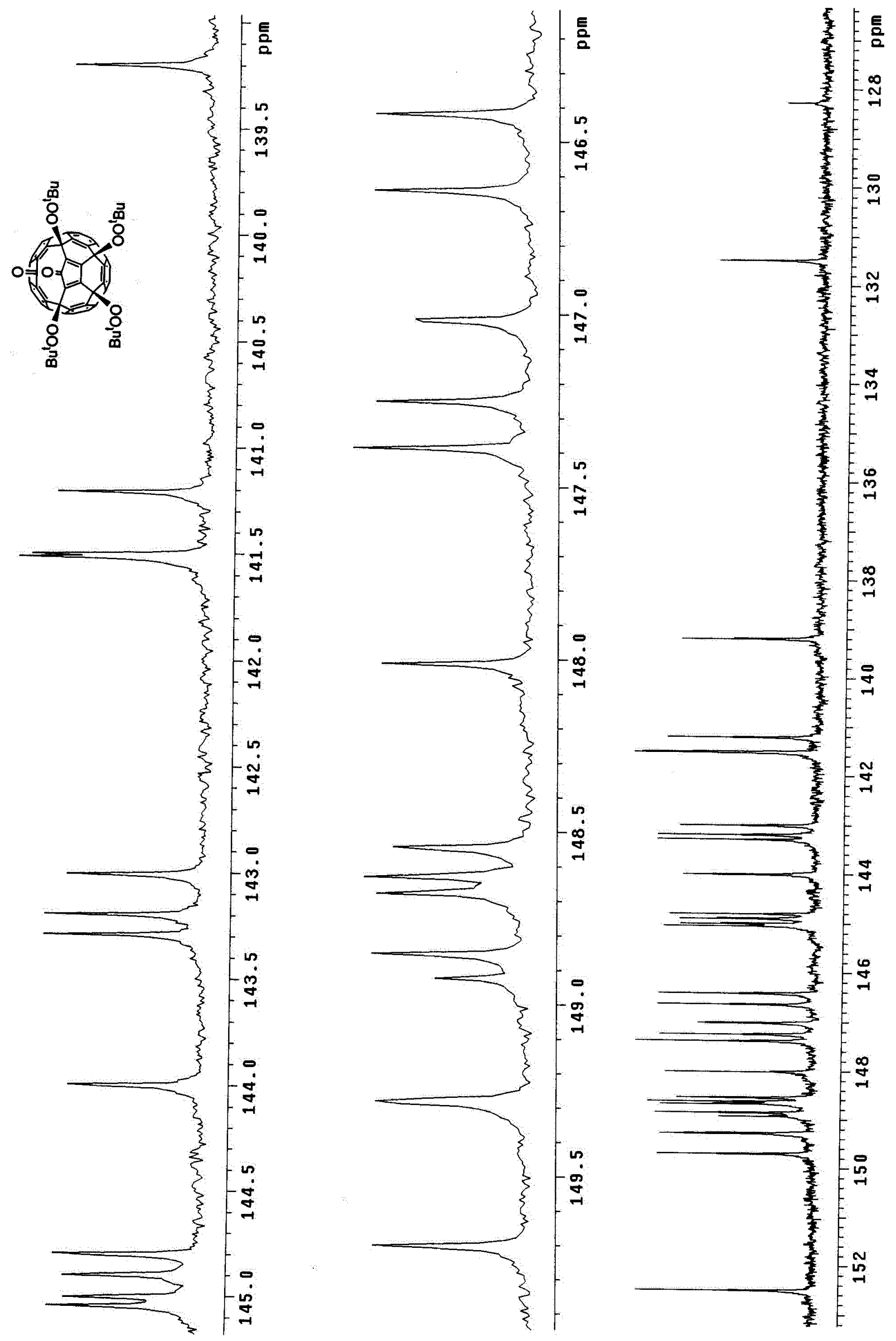



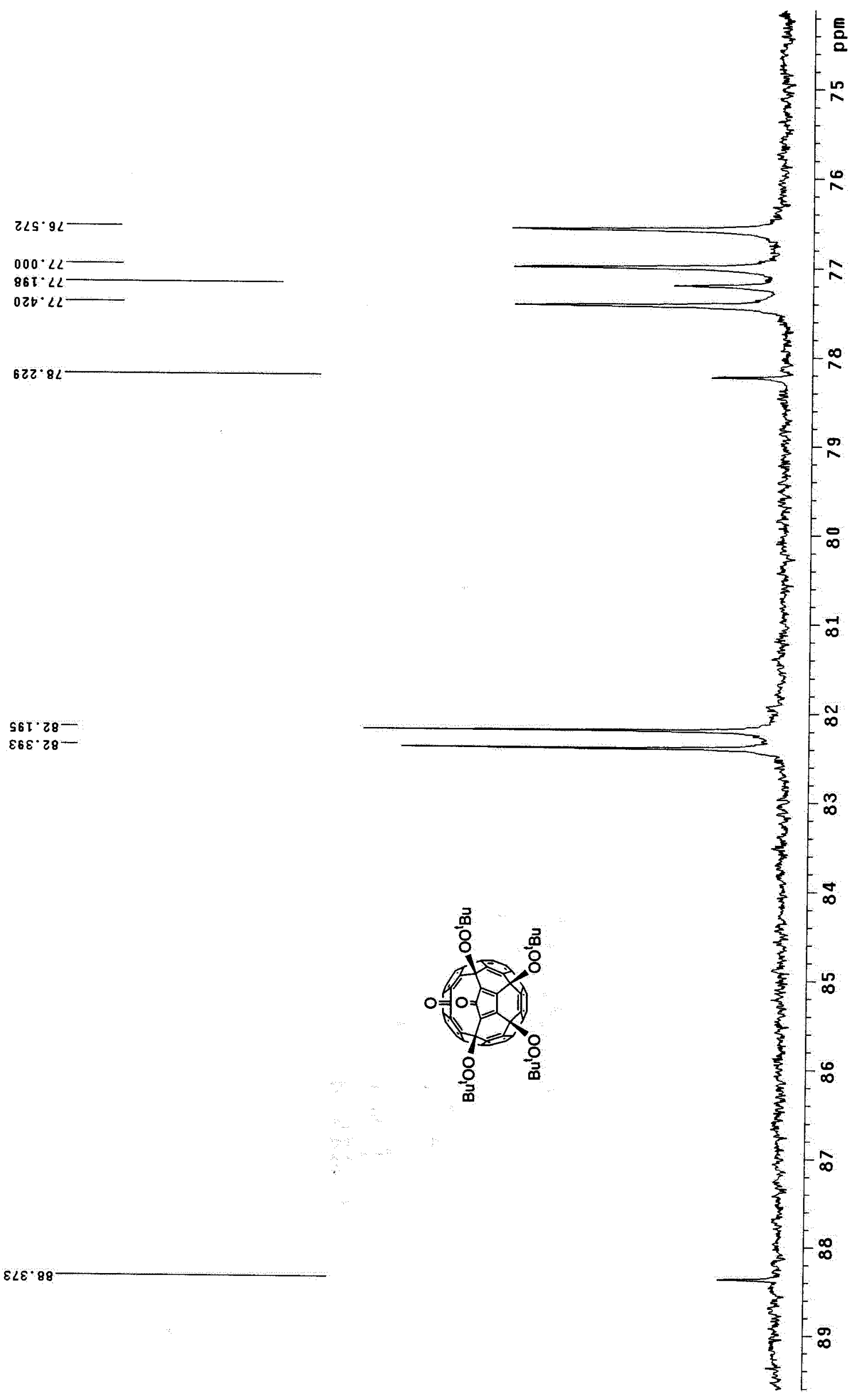

$561 \cdot 28-$

ह6. $28-$

$\varepsilon \angle 8.88$ 


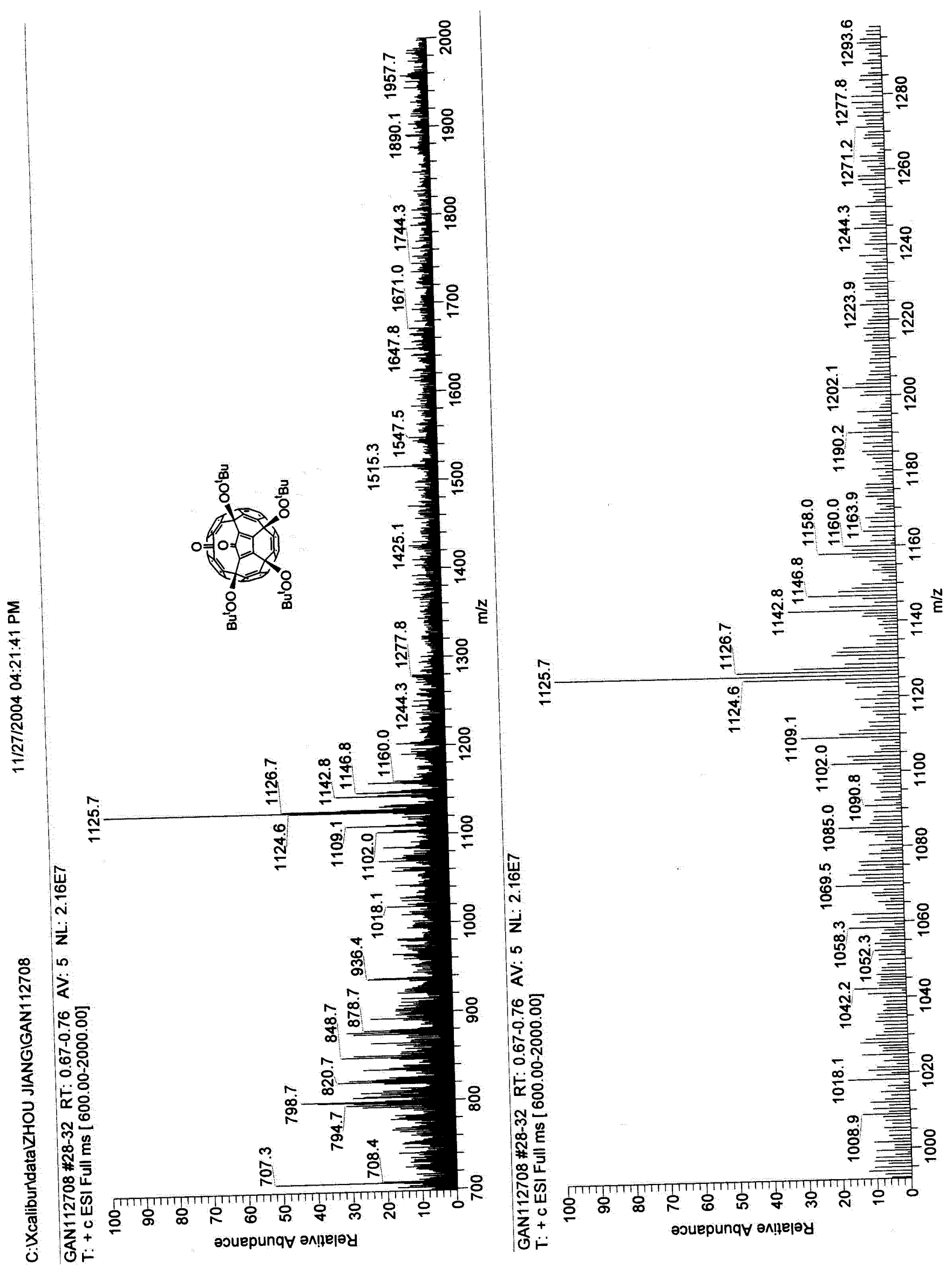




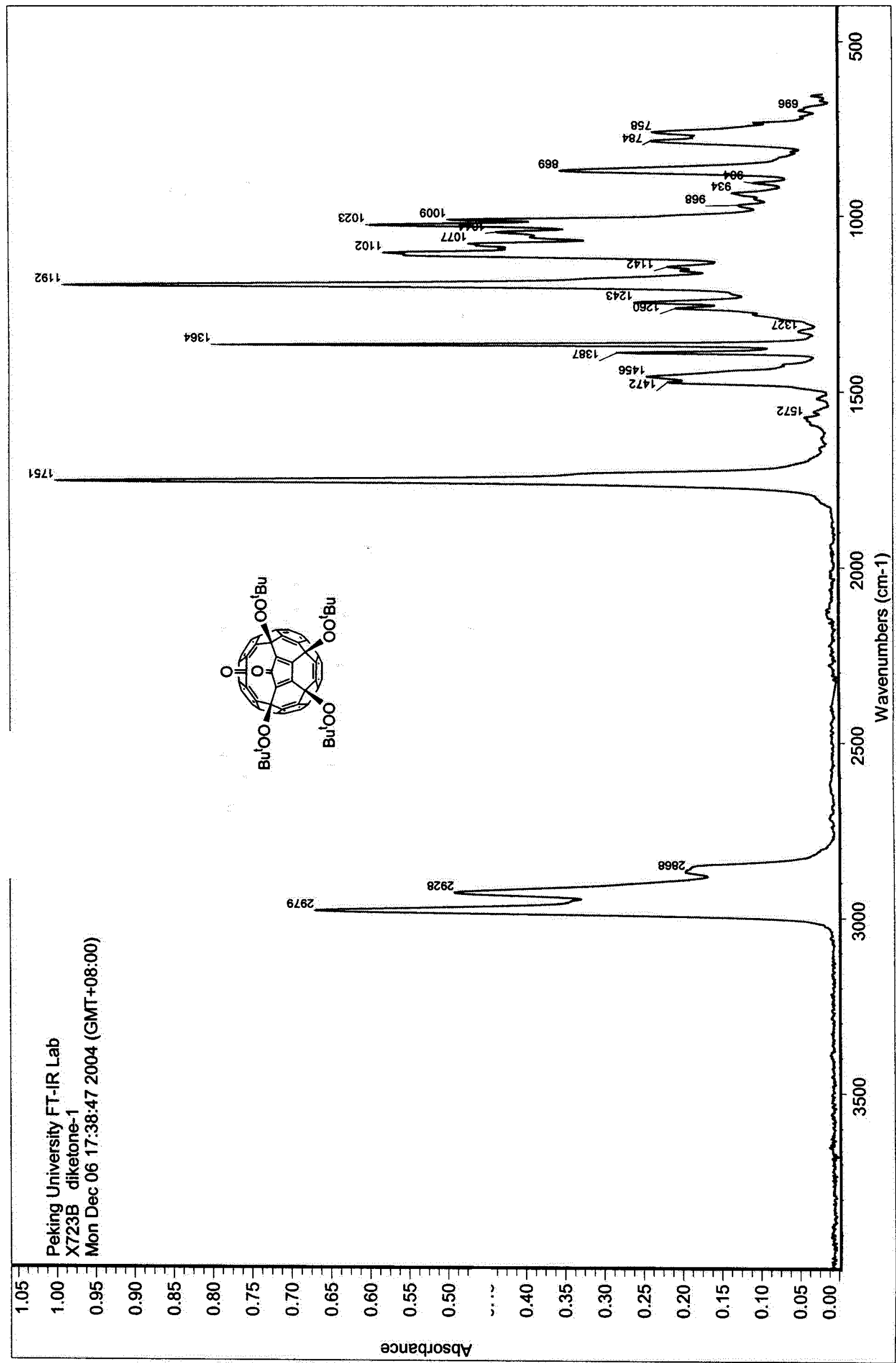


Z8ESट $1-$

S892E $\mathrm{L}$

TOLET

$589 \angle \nabla \cdot-$

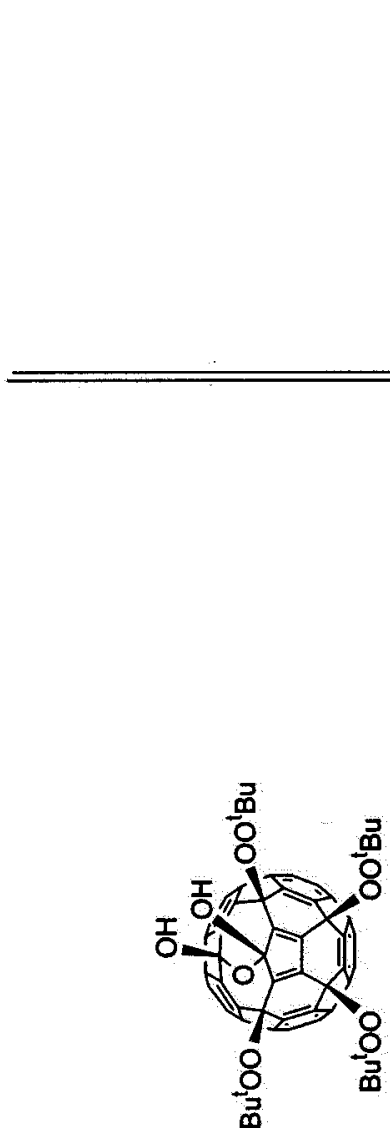

69E IS $レ$

I $9886 \circ$

$0 \varepsilon \angle G E \cdot 己$

HDG8 $\angle$ टG092 ' $L$

$\checkmark 6 E 9 E:$

wdd

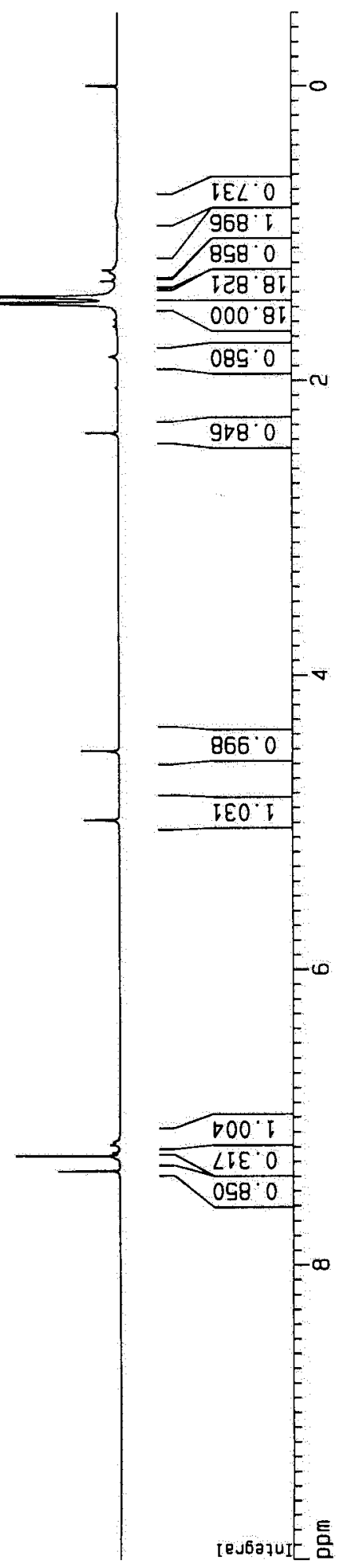




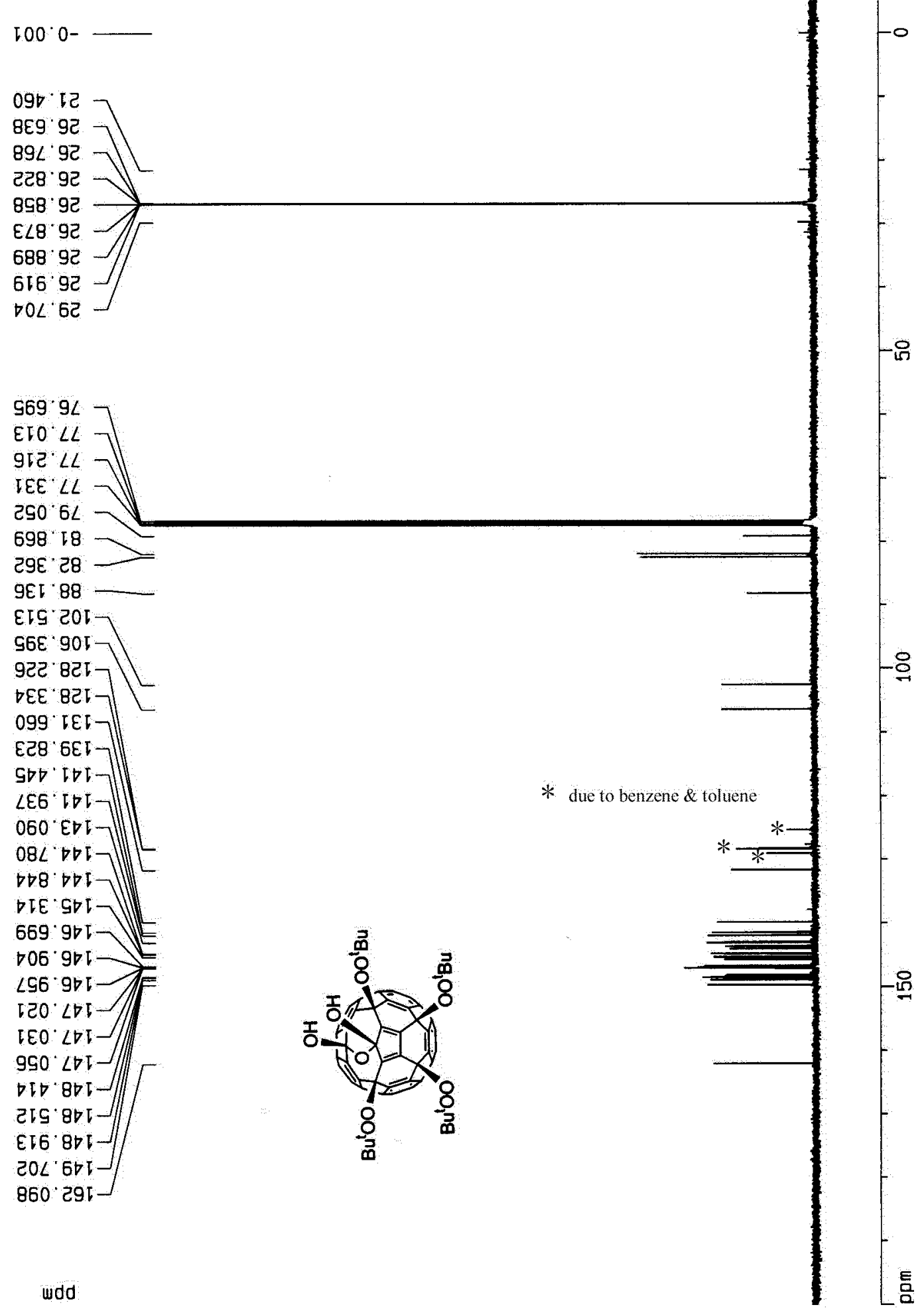




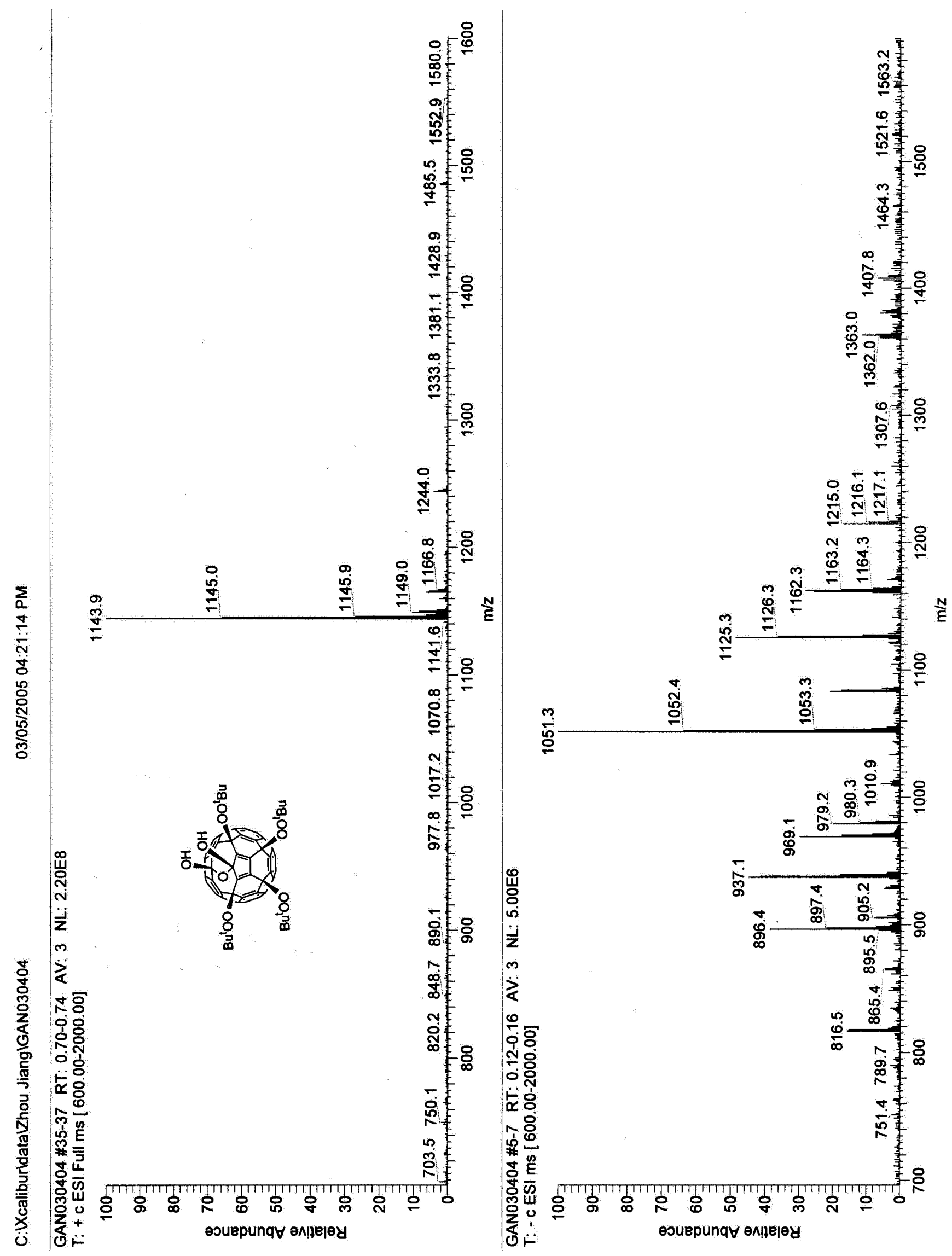


$81026 \%$ 989E6 $0 \longrightarrow$ $\angle$ GGE ${ }^{\circ}$ 89990. $28 \angle \angle D^{\circ} 1-$ $\angle 29 V L^{\circ} \cdot-$

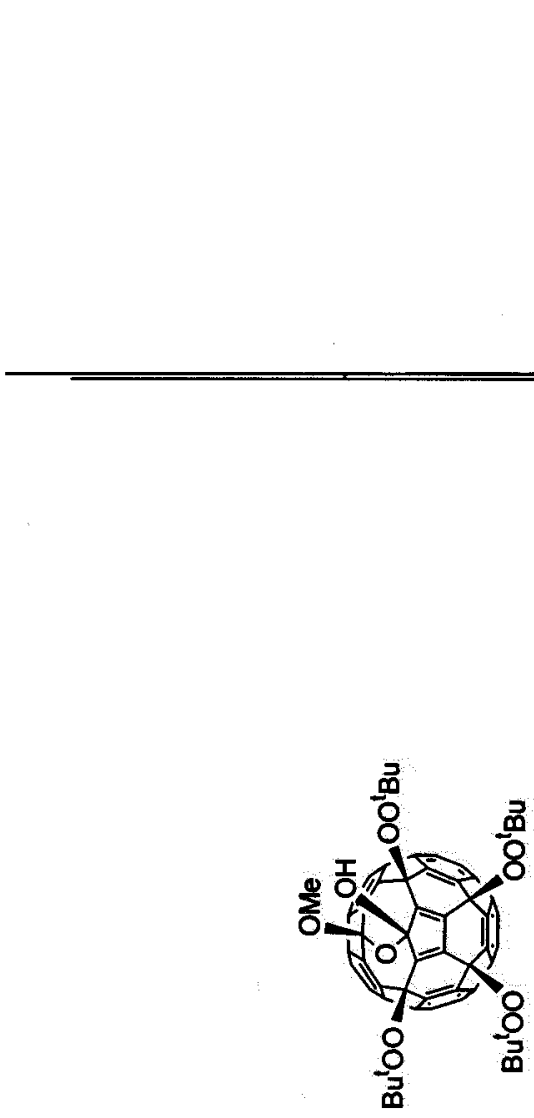

OPGEG $\square-$

$2 \nabla 2 \angle \theta^{\circ} \varepsilon-$

6r092 L

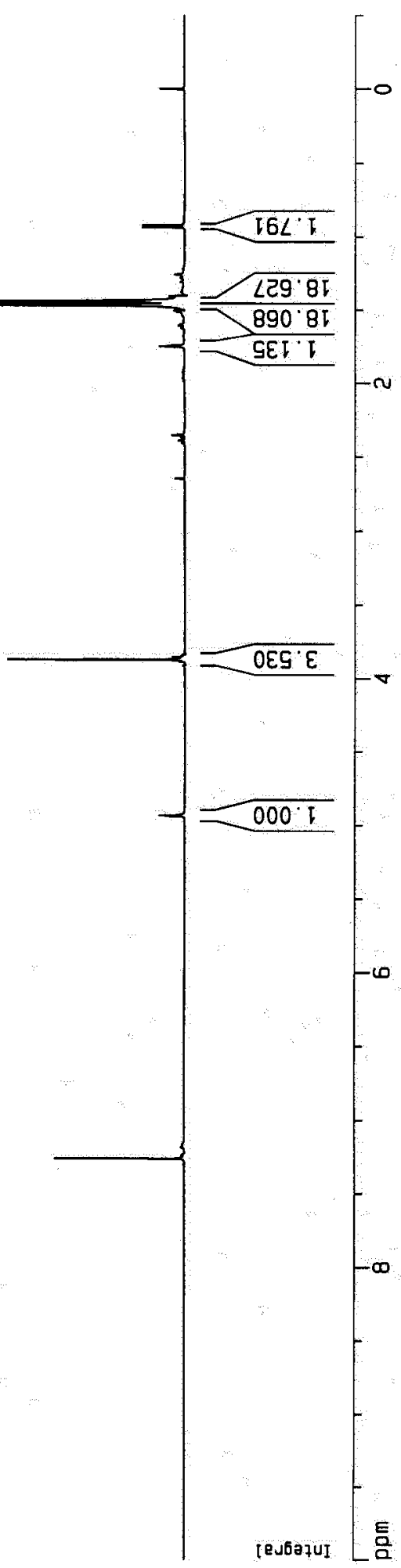




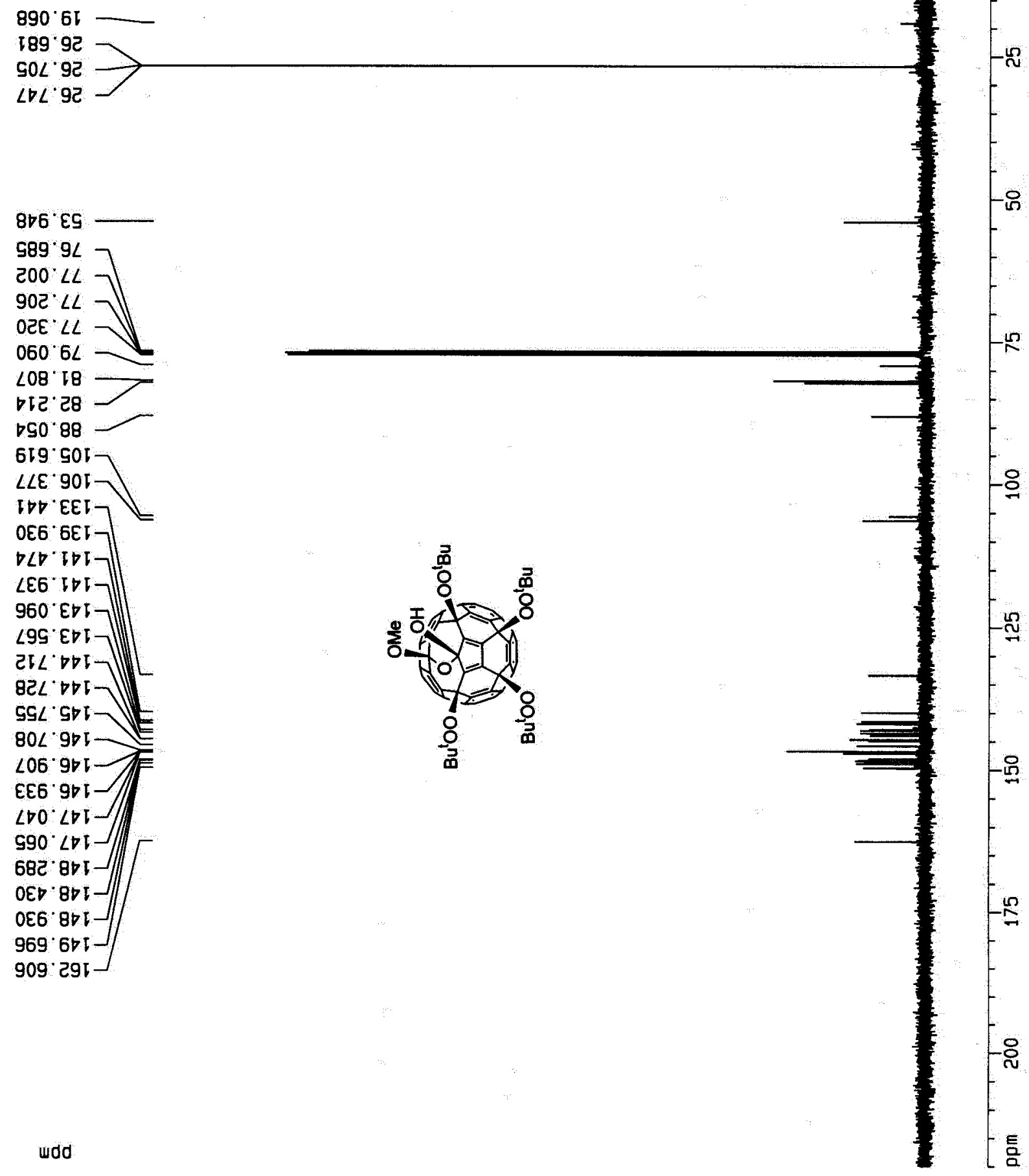




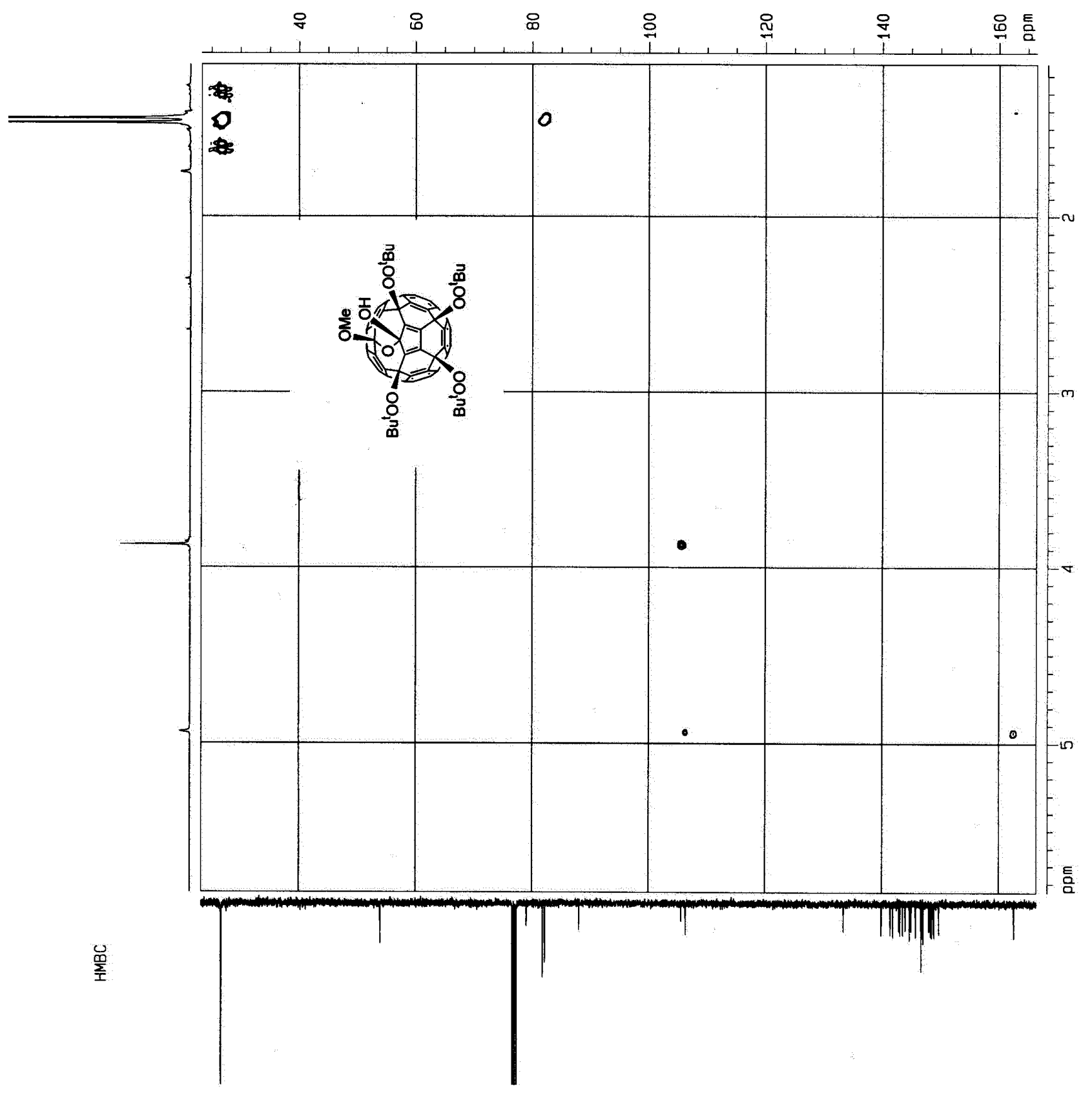




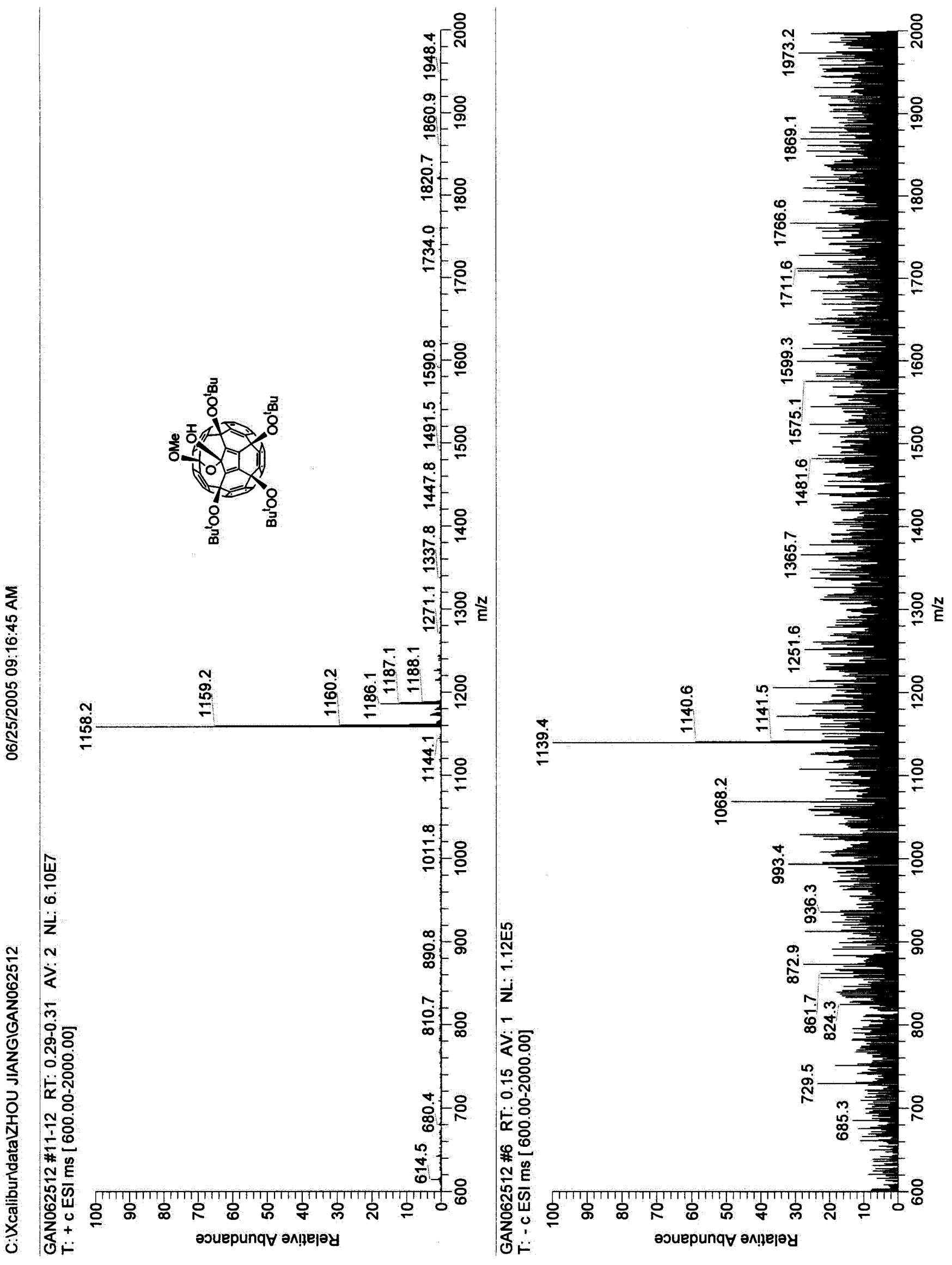




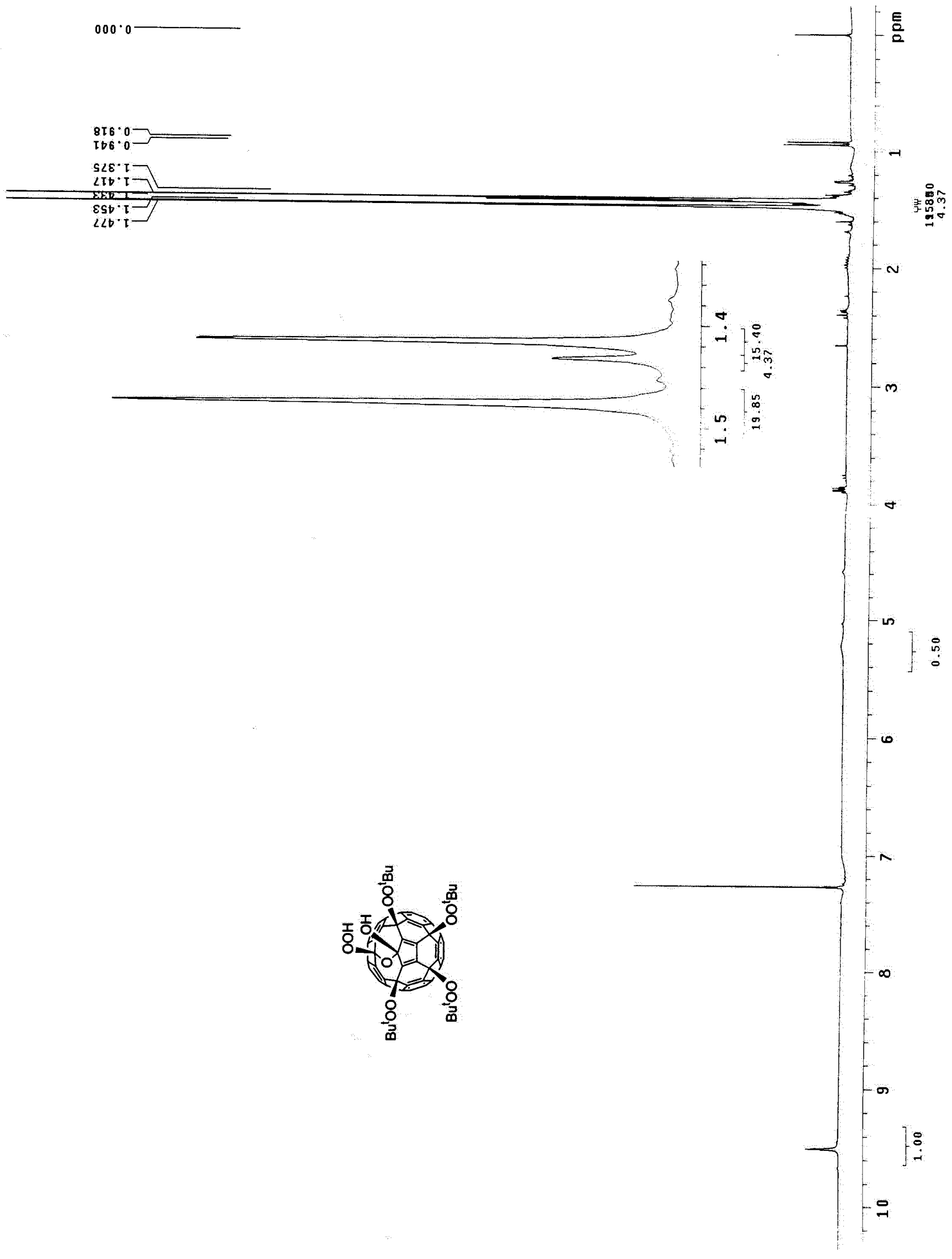




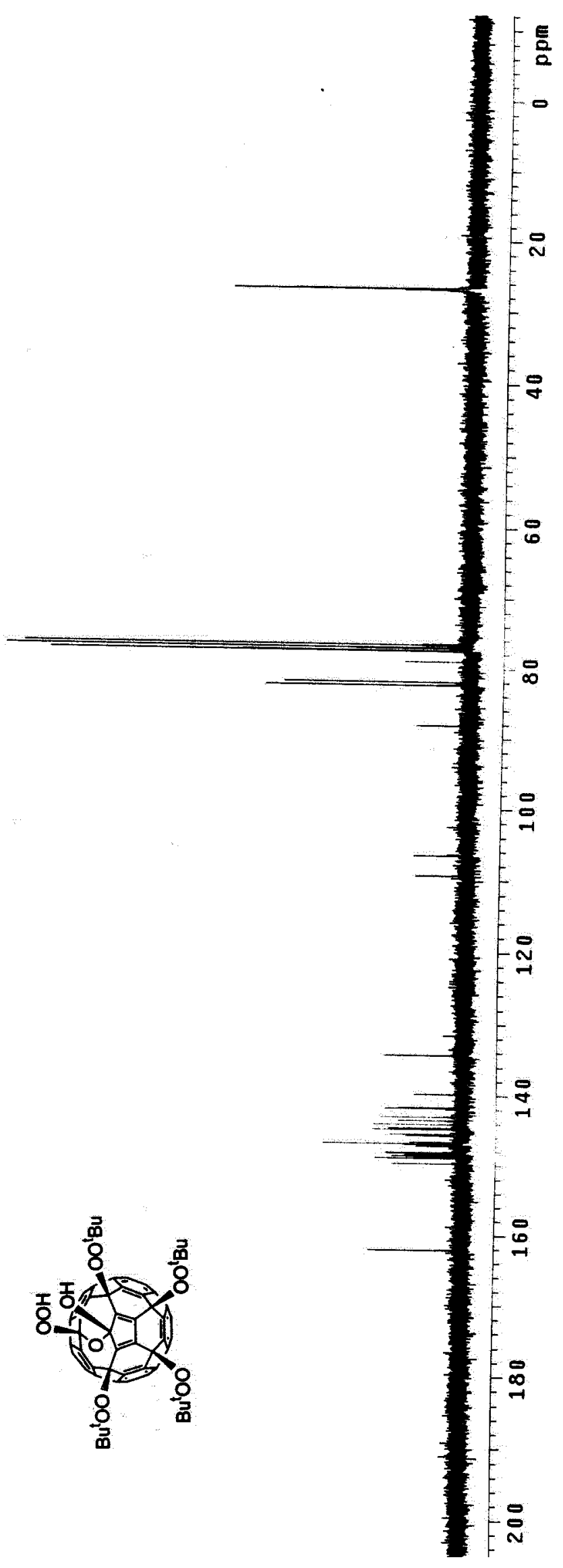




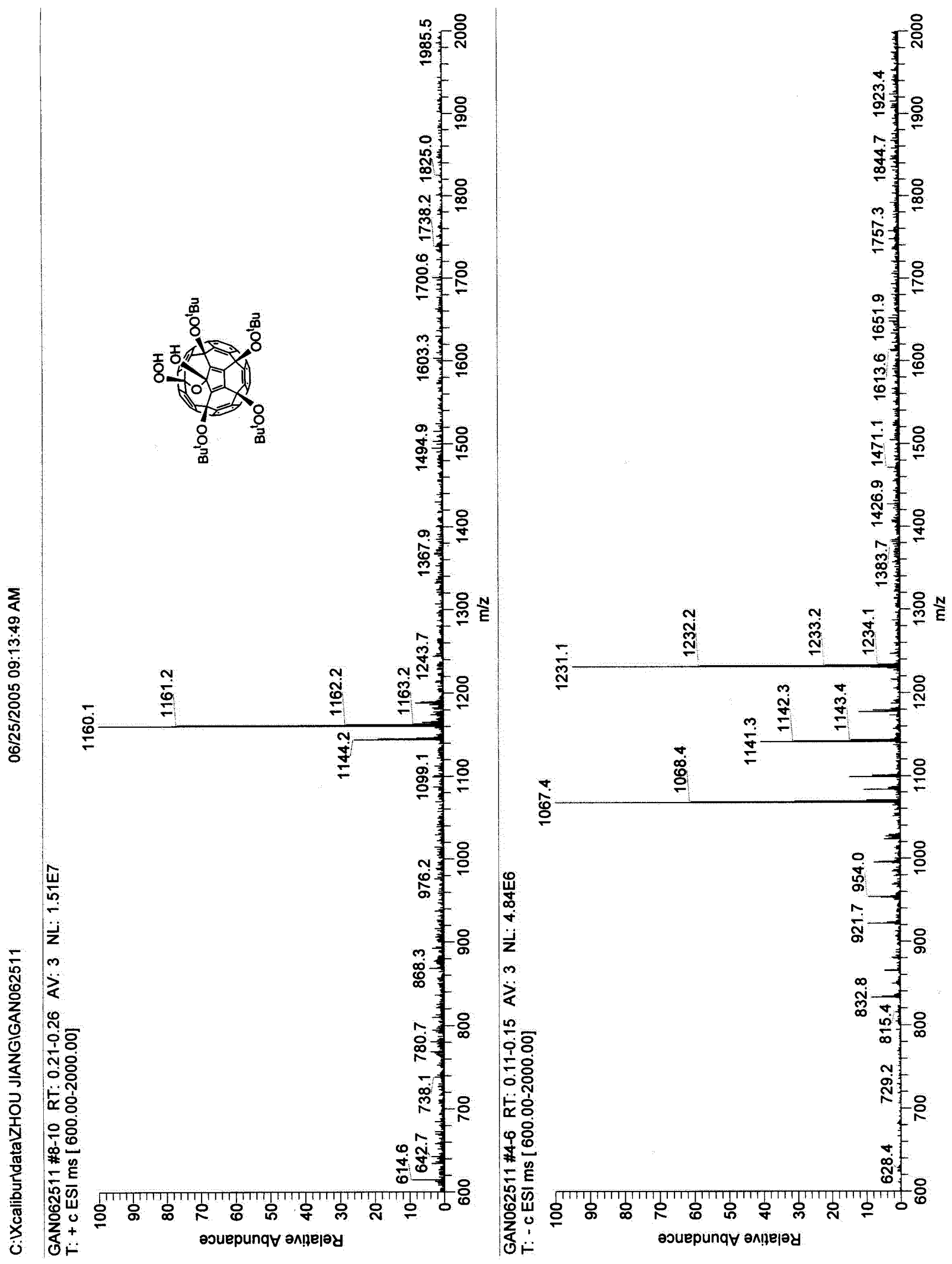




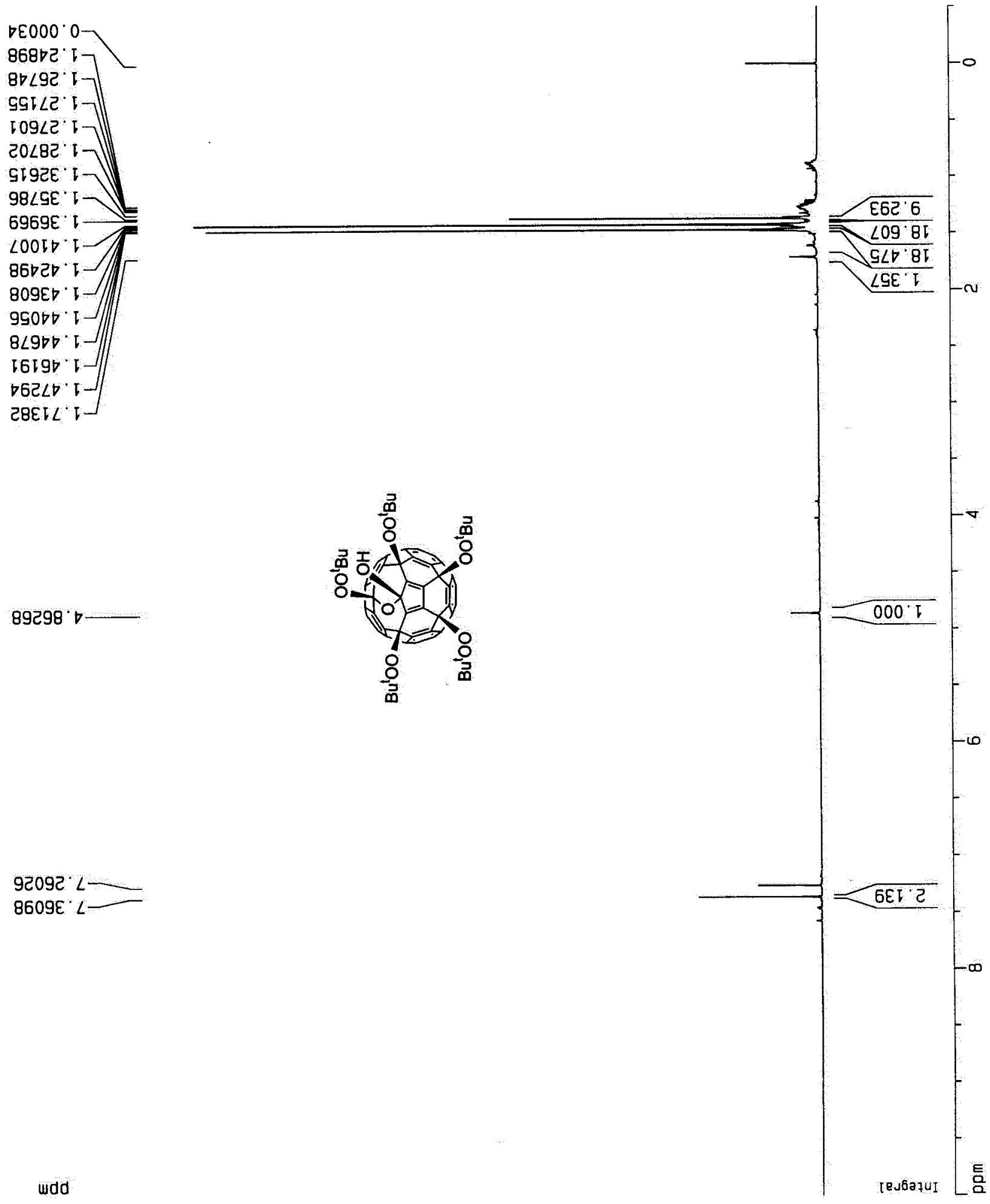




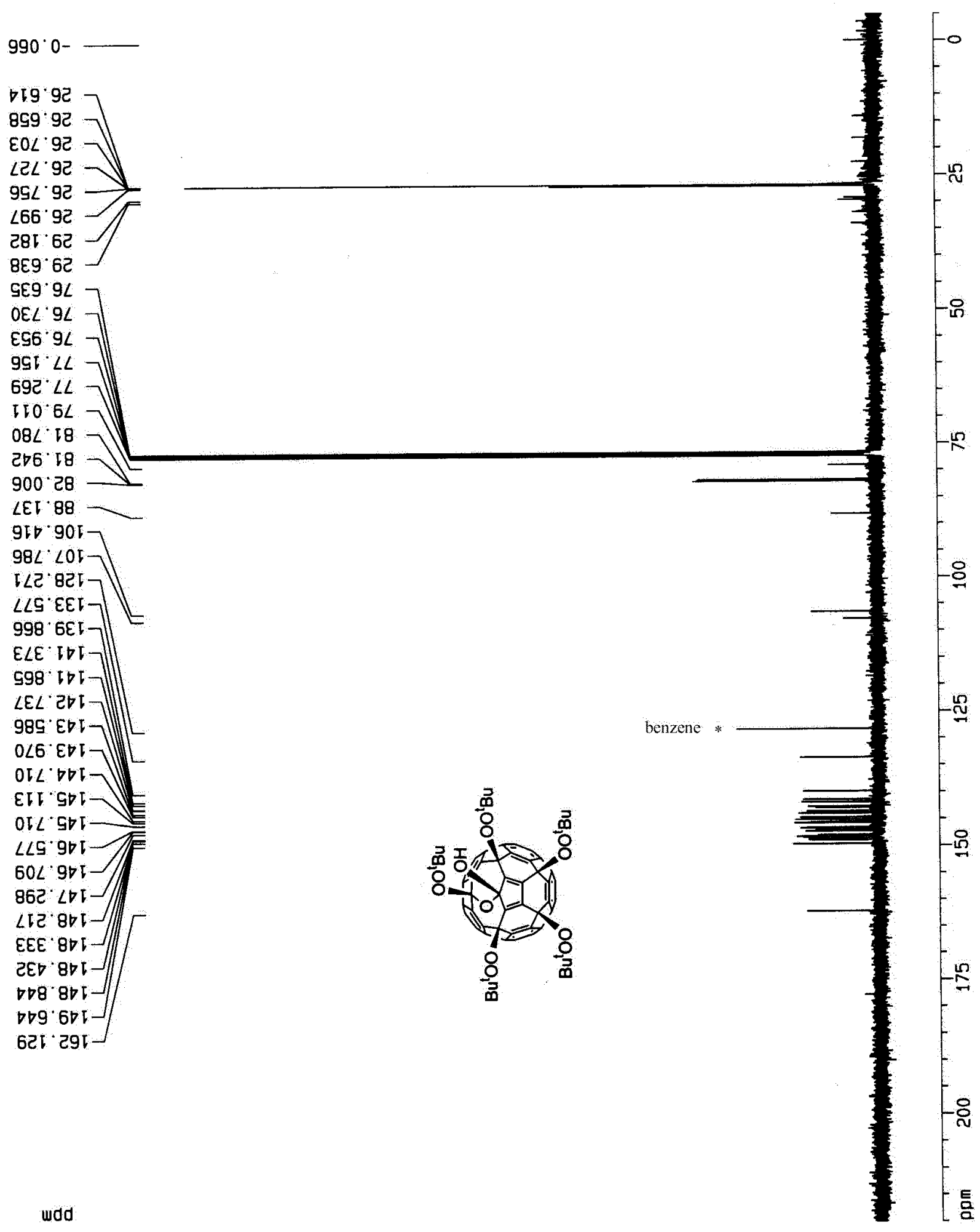




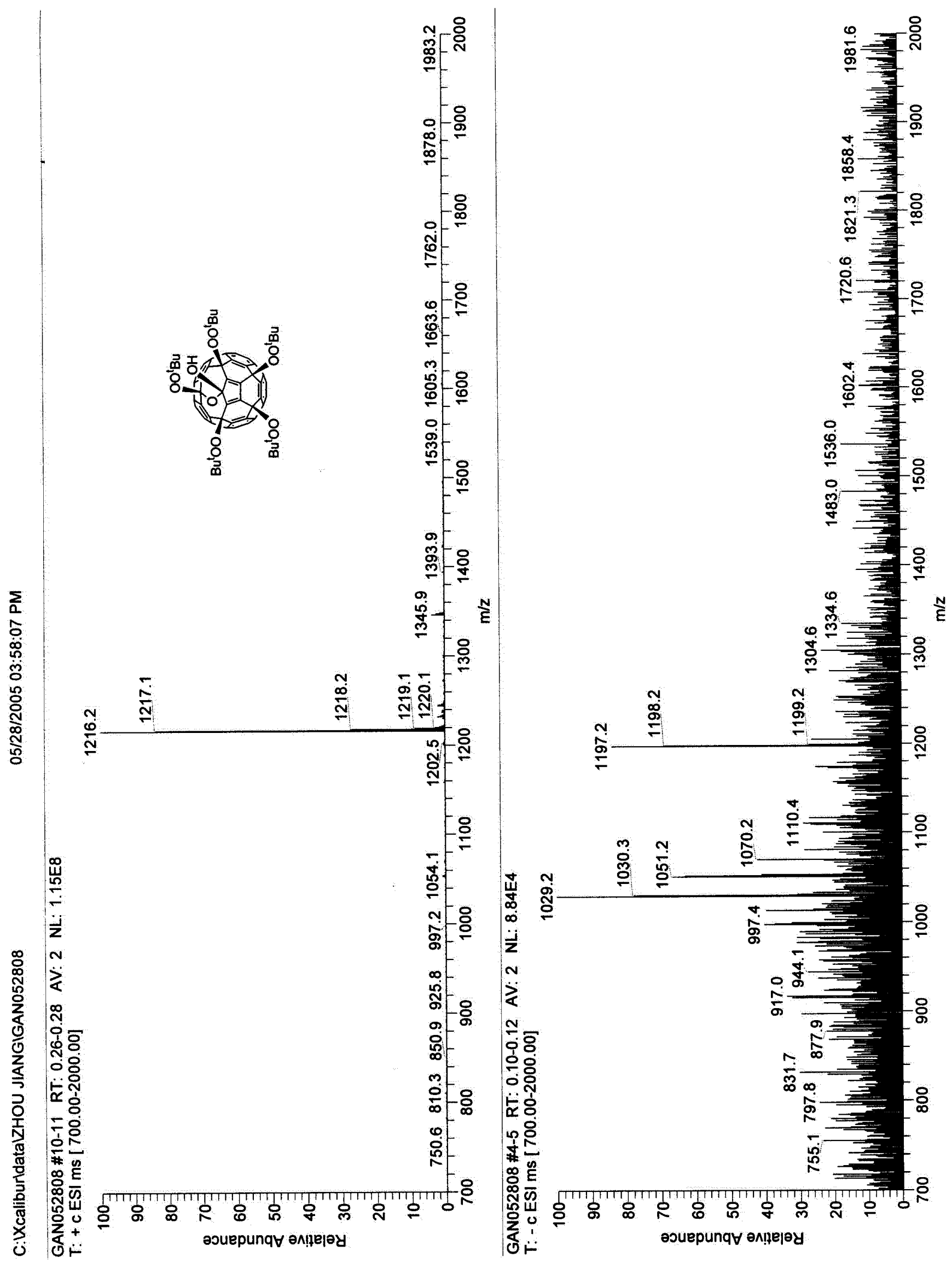

